\title{
Positivity at test of cure following first-line treatment for genital Mycoplasma genitalium: follow-up of a clinical cohort
}

\author{
Zarin Gundevia, ${ }^{1}$ Rosalind Foster, ${ }^{1,2}$ Muhammad Shahid Jamil, ${ }^{2}$ Anna McNulty ${ }^{1,3}$
}

${ }^{1}$ Department of Sexual Health Medicine, Sydney Sexual Health Centre, Sydney,

Australia

${ }^{2}$ The Kirby Institute, University of New South Wales, Sydney, Australia

${ }^{3}$ School of Public Health and Community Medicine, University of New South Wales, Sydney, Australia

\section{Correspondence to} Dr Zarin Gundevia, Level 3, Nightingale Wing, Sydney Sexual Health Centre, Sydney/ Sydney Eye Hospital, Macquarie Street, Sydney, NSW 2000, Australia: zarin@doctors.org.uk

Received 26 March 2014 Revised 14 July 2014 Accepted 19 July 2014 Published Online First 5 August 2014

\section{ABSTRACT}

Objectives To describe antibiotic use for treatment of Mycoplasma genitalium (MG) at an urban sexual health centre in Australia. To describe MG positivity rates in those returning for 1 month test of cure (TOC) following first-line antibiotic treatment for MG.

Methods Retrospective cross-sectional case-note review for all patients diagnosed with MG at Sydney Sexual Health Centre from 2009 to 2013.

Results Two hundred and eighteen MG cases were identified; $66 \%$ were male and $90 \%$ were symptomatic at presentation. Four people did not return for treatment. Azithromycin containing regimens were prescribed as first-line treatment in $88 \%$ of cases; azithromycin $1 \mathrm{~g}$ stat in $75 \%$ of cases and a course of extended azithromycin $1 \mathrm{~g}$ stat plus $500 \mathrm{mg}$ daily for 4 days in $14 \%$ of cases. TOC was performed in $53 \%$ (95\% Cl $46 \%$ to $60 \%)$ of cases and $28 \%(95 \% \mathrm{Cl}$ $20 \%$ to $38 \%$ ) of these cases were MG-positive at TOC. Of those having a MG-positive result at TOC, $26 \%$ received azithromycin $1 \mathrm{~g}$ stat and 33\% received extended azithromycin. Accounting for cases lost to follow-up in azithromycin containing regimens, the positive MG TOC rate was estimated to be between $15 \%$ and $61 \%$.

Conclusions High rates of MG positivity were found in those attending TOC following first-line treatment of MG with azithromycin containing regimens.

\section{INTRODUCTION}

Mycoplasma genitalium (MG) is a sexually transmitted infection that can cause non-gonococcal urethritis (NGU), cervicitis and pelvic inflammatory disease. Limitations exist on MG testing in Australia, as testing facilities are not readily available. Evidence for optimal MG treatment is hampered by lack of testing availability worldwide. Current guidelines in Australia and overseas ${ }^{1-3}$ recommend treating NGU with azithromycin $1 \mathrm{~g}$ stat or doxycyline $100 \mathrm{mg}$ twice daily for 7 days. When first-line treatment fails, all guidelines recommend MG testing where available and treating to cover MG. Some international guidelines recommend an extended course of azithromycin $(500 \mathrm{mg}$ stat and $250 \mathrm{mg}$ daily for 4 days) for first-line treatment of persistent NGU. ${ }^{1}{ }^{3}$ Moxifloxacin is recommended as second-line treatment of persistent NGU. ${ }^{2} 3$

There have been emerging concerns about azithromycin resistance locally. ${ }^{4}$ It was anecdotally noted that many patients were returning positive MG tests of cure (TOCs) at 1 month. This prompted investigating antibiotic use in treatment of $\mathrm{MG}$ and determining MG positivity rates at TOC for all returning patients diagnosed with MG at Sydney Sexual Health Centre (SSHC).

\section{METHODS}

SSHC is a large, urban, Australian sexual health centre. A retrospective review of all patients diagnosed with MG at SSHC was performed, from August 2009 when routine MG testing was introduced, to May 2013. Clinical policy dictates that MG testing at SSHC is performed in heterosexual patients presenting with clinical features of urethritis, cervicitis or pelvic inflammatory disease; men who have sex with men with recurrent NGU and sexual contacts of patients diagnosed with MG. Sampling is achieved via first void urine in men and endocervical swab in women. Specimens are sent to the Institute for Clinical Pathology and Medical Research laboratories at Westmead Hospital, Sydney where MG PCR is performed via inhouse assay to amplify the MgPa gene. Standard first-line treatment of urethritis, cervicitis and sexual contacts of MG is oral azithromycin $1 \mathrm{~g}$ stat. If $\mathrm{MG}$ is detected at first visit but the patient did not receive antibiotics at that time, an extended course of oral azithromycin ( $1 \mathrm{~g}$ stat plus $500 \mathrm{mg}$ daily for 4 days) is given when the patient is recalled for treatment. MG TOC is performed 1 month post treatment. At initial MG diagnosis, patients are made aware of the need to perform TOC and sent a mobile telephone text reminder at 1 month. Patient records for all positive MG diagnoses were manually reviewed to extract demographic and clinical information. Data were entered onto a Microsoft Excel spreadsheet and analysed using STATA V.12 (StataCorp, College Station, Texas, USA). Frequencies, 95\% CIs and $\mathrm{p}$ values were reported. OR was calculated to determine associations between presence of symptoms and positive TOC. Ethics approval was obtained via the South Eastern Sydney Local Health District Human Research Ethics Committee (approval number 08/223).

\section{RESULTS}

The study identified 218 MG cases and 144 (66\%) were male. The mean age was 30 years (range: 15-57 years). At initial presentation, 196 (90\%) were symptomatic. Eight (4\%) patients presented as sexual contacts of patients diagnosed with MG. Recent heterosexual activity only was reported in 182 (83\%) cases. Overall, 193 (89\%) patients 
reported inconsistent condom use for vaginal or anal sex in the preceding 3 months. Of all patients having microscopy at first presentation, $57 \%(36 / 63)$ of women and $83 \%(102 / 123)$ of men had significant findings as defined by clinic protocol $(>30$ polymorphs/high powered field in cervical gram staining and $>5$ polymorphs/high powered field in male urethral gram staining). Four people did not return to receive treatment at SSHC.

Oral azithromycin $1 \mathrm{~g}$ was the most commonly prescribed first-line treatment $(75 \%, 95 \%$ CI $68 \%$ to $80 \%)$. TOC was performed in 113 (53\%, 95\% CI 46\% to 60\%) patients; 73 (51\%, $95 \%$ CI $43 \%$ to $59 \%)$ men and $40(56 \%, 95 \%$ CI $45 \%$ to $68 \%)$ women $(\mathrm{p}=0.47)$. Overall, $32(28 \%$, 95\% CI $20 \%$ to $38 \%)$ patients had a positive MG at TOC. Among those who received azithromycin treatment, the 1 month MG TOC positivity rate was $27 \%$ (95\% CI $19 \%$ to $37 \%)$. A summary of these results is presented in figure 1.

The proportion of positive TOCs at 1 month did not differ significantly between men and women (26\% (95\% CI 16\% to $36 \%)$ vs $33 \%$ (95\% CI $18 \%$ to $47 \%), \mathrm{p}=0.47$ ) or between those with and without symptoms at baseline $(28 \%(95 \% \mathrm{CI}$ $19 \%$ to $37 \%$ ) vs $33 \%$ (95\% CI $2.5 \%$ to $64 \%$ ), $\mathrm{p}=0.73$ ). Regarding azithromycin-containing regimes, if cases lost to follow-up had negative TOCs, the positive TOC rate would be $28 / 189(15 \%, 95 \%$ CI $10 \%$ to $21 \%)$. If cases lost to follow-up had positive TOCs, the positive TOC rate would be $115 / 189$ (61\%, 95\% CI 54\% to 68\%).

Second-line antibiotics were administered to patients based on clinical findings and/or positive TOC. A second-line antibiotic regime was prescribed for 63 patients. Of these patients, 43 (68\%, 95\% CI 55\% to 79\%) were symptomatic. Second-line regimes comprised of azithromycin $1 \mathrm{~g}$ stat for 10 patients, extended azithromycin for 15 patients, doxycycline for
2 patients and moxifloxacin for 36 patients. Despite SSHC policy not requiring TOC following second-line treatment of MG, 14 (22\%) patients had subsequent TOC 1 month after the second antibiotic course. One patient (7\%, 95\% CI $0.2 \%$ to $34 \%$ ) had a positive subsequent TOC despite an initial course of doxycycline and a subsequent course of moxifloxacin after the first positive TOC.

Following first-line treatment with azithromycin, 87 (46\%) patients were lost to follow-up. No statistically significant differences existed between those lost to follow-up and those engaged in care regarding: gender (69\% male (95\% CI 59\% to $79 \%)$ vs $62 \%$ male $(95 \%$ CI $52 \%$ to $71 \%), \mathrm{p}=0.30)$; mean age ( 29 years (95\% CI 27 to 31 ) vs 30 years (95\% CI 29 to 32 ), $\mathrm{p}=0.28$ ); symptoms at presentation (89\% (95\% CI $82 \%$ to $95 \%$ ) vs $92 \%$ (95\% CI $87 \%$ to $97 \%), \mathrm{p}=0.39$ ); recent same sex partner $(5 \%$ (95\% CI $0.2 \%$ to $9 \%$ ) vs $11 \%$ (95\% CI $5 \%$ to $17 \%), p=0.12$ ) and consistent condom use in last 3 months (6\% (95\% CI 1\% to $11 \%$ ) vs $13 \%$ (95\% CI $6 \%$ to $19 \%), \mathrm{p}=0.10)$.

Of the 113 patients attending TOC, 36 (32\%, 95\% CI 23\% to $41 \%$ ) reported symptoms in the 4 weeks after initial treatment. Positive TOCs were found in 17 (47\%, 95\% CI 30\% to $65 \%)$ of these patients. Of the $77(68 \%)$ patients reporting no genital symptoms in the 4 weeks after initial treatment, 15 (19\%, 95\% CI $11 \%$ to $30 \%)$ had a positive TOC. Having genital symptoms at some point during the 4 weeks after initial treatment was associated with a positive $\mathrm{MG}$ result at TOC (OR $3.70,95 \%$ CI 1.6 to $8.8, \mathrm{p}=0.003)$.

\section{DISCUSSION}

This study demonstrates high rates of MG positivity at $27 \%$ in patients attending 1 month TOC following azithromycin treatment. Recent existing data for MG shows eradication rates of

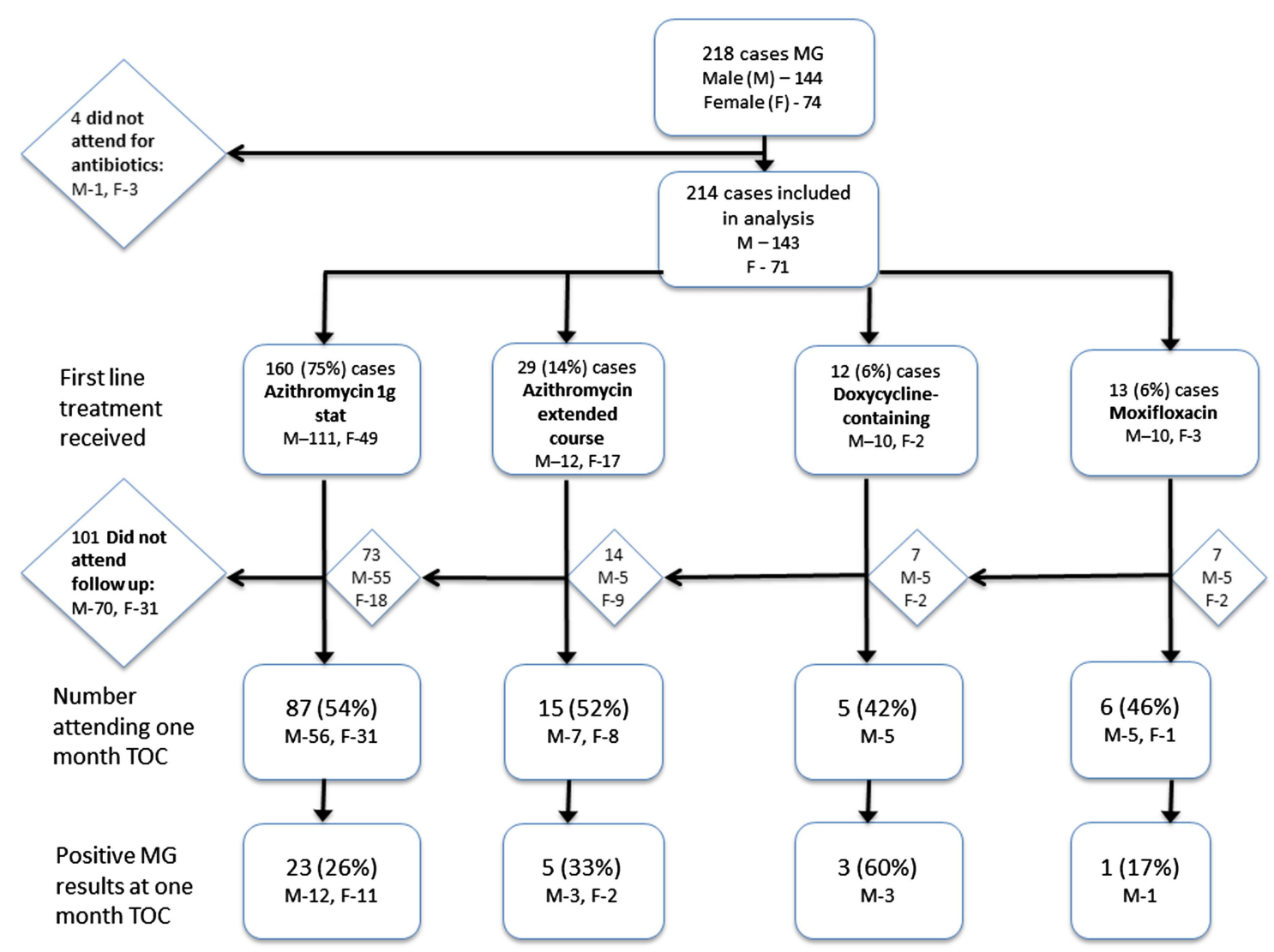

Figure 1 Attendance, antibiotic use and MG positivity at one month TOC in the study population. 
40-91\% with first-line azithromycin stat therapy ${ }^{5} 6$ and 78-99\% with extended azithromycin. ${ }^{6} 7$ Azithromycin $1 \mathrm{~g}$ stat eradication rates appear to be declining over time. ${ }^{5}$ One possible explanation is macrolide mutation associated resistance. Recent Australian data have shown induced macrolide resistance of 25$55 \%$ following azithromycin $1 \mathrm{~g}$ stat. ${ }^{48}$ Treatment failure may account for some MG-positive TOC results, however our study findings do not distinguish between treatment failure and re-infection.

The study data demonstrate similar rates of MG positivity at TOC with extended and stat azithromycin and no statistically significant difference in treatment outcomes. Numbers are small and should be interpreted with caution. Jernberg et al demonstrated that extended azithromycin was no more efficacious than a single dose. ${ }^{7}$

This study demonstrates high levels of MG positivity following doxycycline and supports previously published data that demonstrated a $55-83 \%$ failure rate using doxycycline as firstline treatment of MG. ${ }^{5} 6$

One case remained MG-positive despite a course of moxifloxacin. Published studies demonstrate MG eradication rates with first-line moxifloxacin $400 \mathrm{mg}$ for 10 days to be $100 \%$ effective $^{7}$ and $70-100 \%$ efficacy as second-line treatment. ${ }^{4} 7$ Local research describes clinical and microbiological moxifloxacin treatment failure due to fluoroquinolone resistance-associated mutations, including baseline resistance detected in approximately $20 \%$ of cases. ${ }^{4}$

In this study, the presence of symptoms after treatment predicts a positive TOC. The loss to follow-up rate was $47 \%$. It could be postulated that people whose symptoms had disappeared post treatment would be less likely to return for TOC and that those returning for TOC may therefore not be representative of the entire treated population. We found that presence of symptoms in the 4 weeks post treatment significantly affected the 1 month TOC MG result. This is in line with findings of Bradshaw et al, ${ }^{9}$ which showed that people with positive MG TOC results are significantly more likely to report persistence of symptoms at 1 month than people with MG negative TOCs.

Limitations can exist with retrospective studies due to bias, potential for missing data and difficulties with case ascertainment. In this report, such bias should be minimal because all data are routinely collected and entered prospectively as standard clinic practice, including diagnostic clinical code entry with quality control checking. The high number of cases lost to follow-up in this study can introduce bias and should be considered when interpreting TOC results. The data does not determine whether failures were due to resistance or re-infection. Most cases received azithromycin $1 \mathrm{~g}$ stat. This affects statistical comparison with other treatments due to small numbers in other groups. Current clinic policy means the majority of patients undergoing MG testing are symptomatic therefore this population may not be representative of all people infected with MG.

Nevertheless, the findings of this study add to emerging international evidence that azithromycin may be a less effective firstline therapy for the treatment of MG than current guidelines suggest.

\section{Key messages}

Azithromycin containing regimens were most commonly prescribed as first-line treatment for Mycoplasma genitalium (MG).

- A high rate of MG positivity (27\%) was found in those attending 1 month test of cure (TOC) following treatment with azithromycin containing regimens.

- In the study population, presence of symptoms in the 4 weeks post initial treatment was associated with MG positivity at 1 month TOC.

\section{Handling editor Jackie A Cassell}

Acknowledgements The authors thank Dr Neisha Jeoffreys, Senior Hospital Scientist, CIDMLS, Pathology West-ICPMR for providing information on the PCR assay for MG, performed at ICPMR.

Collaborators ZG; RF; MSJ; AMMcN.

Contributors All authors substantially contributed to the conception or design of the work, or the acquisition, analysis or interpretation of data. AMMCN, RF and ZG conceived and designed the study. ZG acquired the data. ZG, RF and MSJ analysed and interpreted the data. RF and ZG drafted the work. All authors revised it critically for important intellectual content and give final approval of the version published. AMMCN is the guarantor. All authors agree to be accountable for all aspects of the work in ensuring that questions related to the accuracy or integrity of any part of the work are appropriately investigated and resolved. All authors had full access to the data and can take responsibility for its accuracy and integrity.

Competing interests ZG received funding from Viiv to attend the Australian HIV and AIDS Conference in 2013.

Ethics approval South Eastern Sydney Local Health District Human Research Ethics Committee.

Provenance and peer review Not commissioned; externally peer reviewed.

\section{REFERENCES}

1 Antibiotic Expert Group. Therapeutic guidelines: antibiotic. Version 14. Melbourne: Therapeutic Guidelines Limited, 2010.

2 Workowski K; Centers for Disease Control and Prevention. Sexually Transmitted Diseases Treatment Guidelines, 2010. MMWR Recomm Rep 2010;59(RR-12):1-110. http://www.cdc.gov/mmwr/preview/mmwrhtml/rr5912a1.htm (accessed 6 Mar 2014).

3 Shamanesh M, Moi H, Lassau F, et al. 2009 European Guideline on the management of non-gonococcal urethritis. Int J STD AIDS http://www.iusti.org/ regions/europe/pdf/2009/euro_ngu_0409.pdf (accessed 6 Mar 2014).

4 Couldwell DL, Tagg KA, Jeoffreys NJ, et al. Failure of moxifloxacin treatment in Mycoplasma genitalium infections due to macrolide and fluoroquinolone resistance. Int J STD AIDS 2013;24:822-8.

5 Manhart LE, Gillespie CW, Lowens MS, et al. Standard treatment regimens for nongonococcal urethritis have similar but declining cure rates: a randomized controlled trial. Clin Infect Dis 2013;56:934-42.

6 Anagrius $C$, Loré $B$, Jensen JS. Treatment of Mycoplasma genitalium. Observations from a Swedish STD clinic. PLOS ONE 2013;8:e61481.

7 Jernberg E, Moghaddam A, Moi H. Azithromycin and moxifloxacin for microbiological cure of Mycoplasma genitalium infection: an open study. Int J STD AIDS 2008; 19:676-9.

8 Twin J, Jensen JS, Bradshaw CS, et al. Transmission and selection of macrolide resistant Mycoplasma genitalium infections detected by rapid high resolution melt analysis. PLOS ONE 2012;7:e35593.

9 Bradshaw CS, Chen MY, Fairley CK. Persistence of Mycoplasma genitalium following azithromycin therapy. PLOS ONE 2008;3:e3618. 